\title{
Generalizing a Data Analysis Pipeline in the Cloud to Handle Diverse Use Cases in NASA's EOSDIS
}

Christopher Lynnes*, NASA/Goddard Space Flight Center Rahul Ramachandran*, NASA/Marshall Space Flight Center 
- Actively manages NASA's Earth science data as a national asset: satellite, airborne, and in situ

- Develops capabilities to support rigorous science research

- Processes instrument data to create high quality long-term Earth science data records.

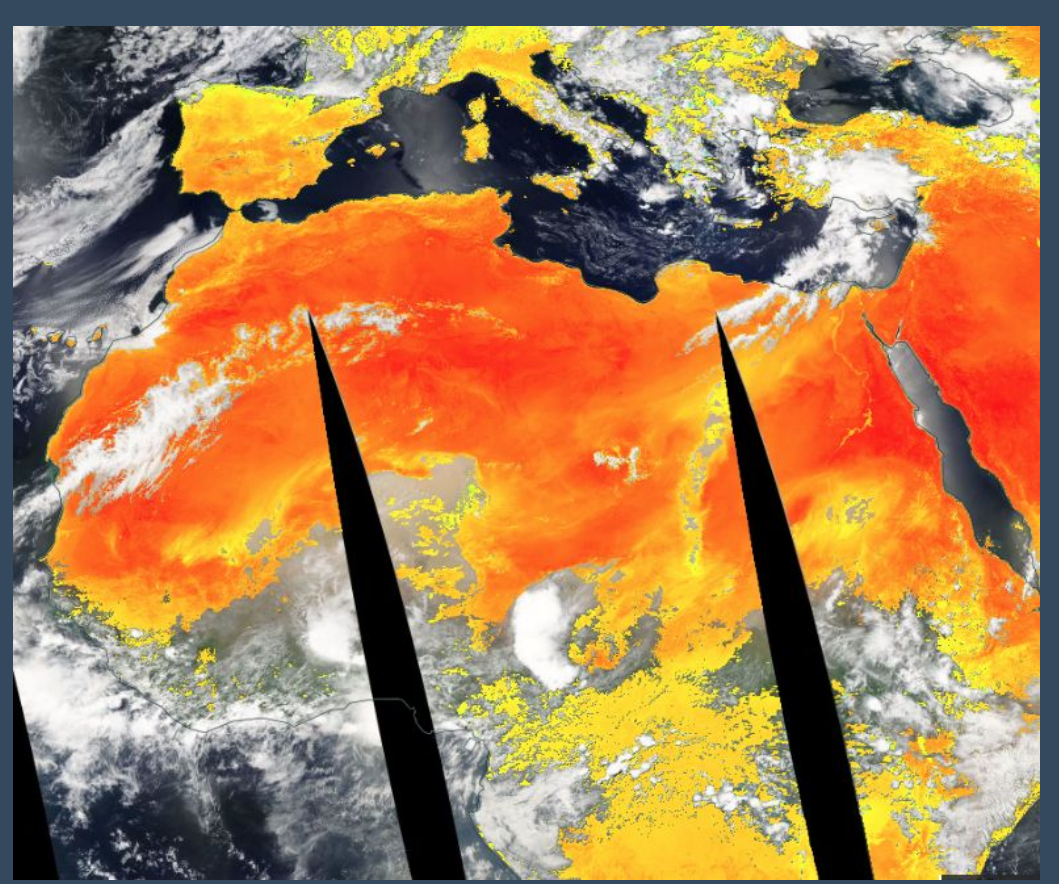

Land Surface Temperature on a base of Corrected Reflectance from Aqua Moderate Resolution Imaging Spectroradiometer, 16 Jun 2018 

Information System

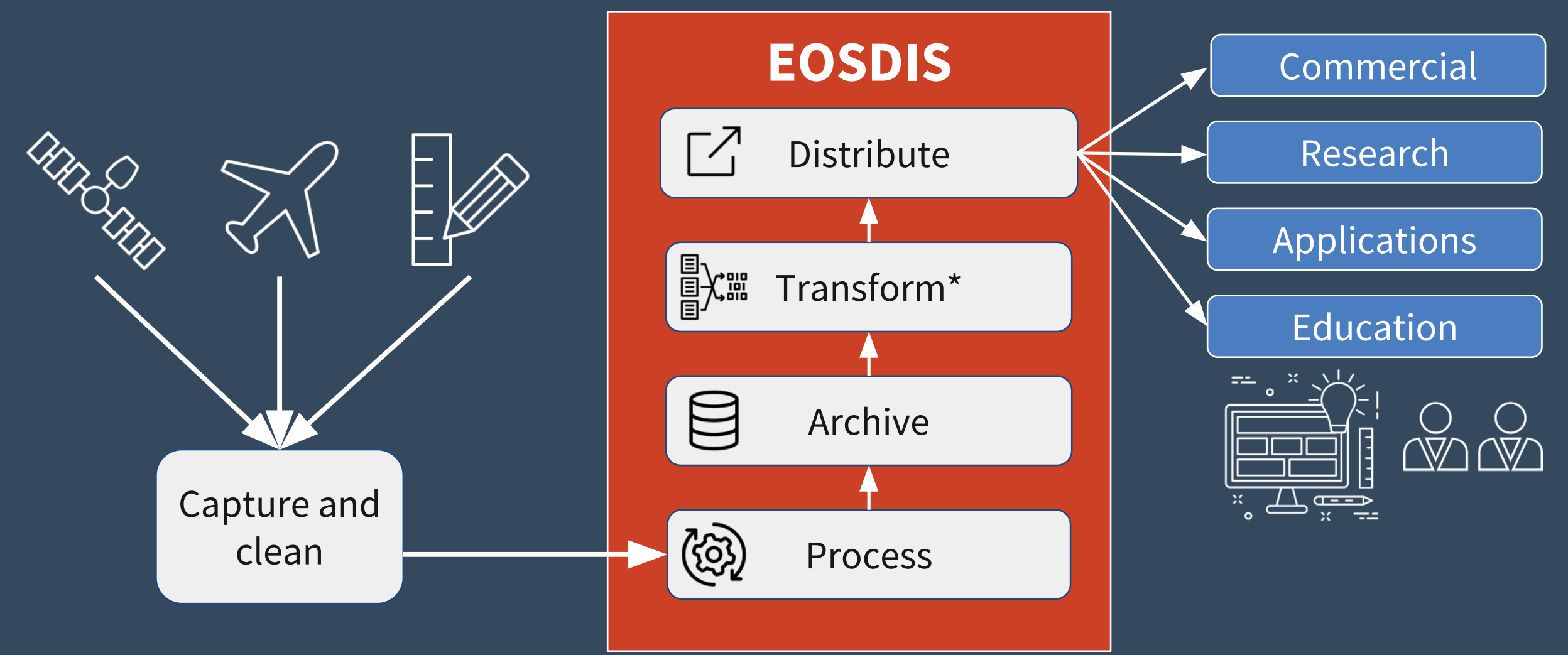


Projected Data Volumes

0
0
$\frac{1}{2}$
$\frac{0}{0}$
0

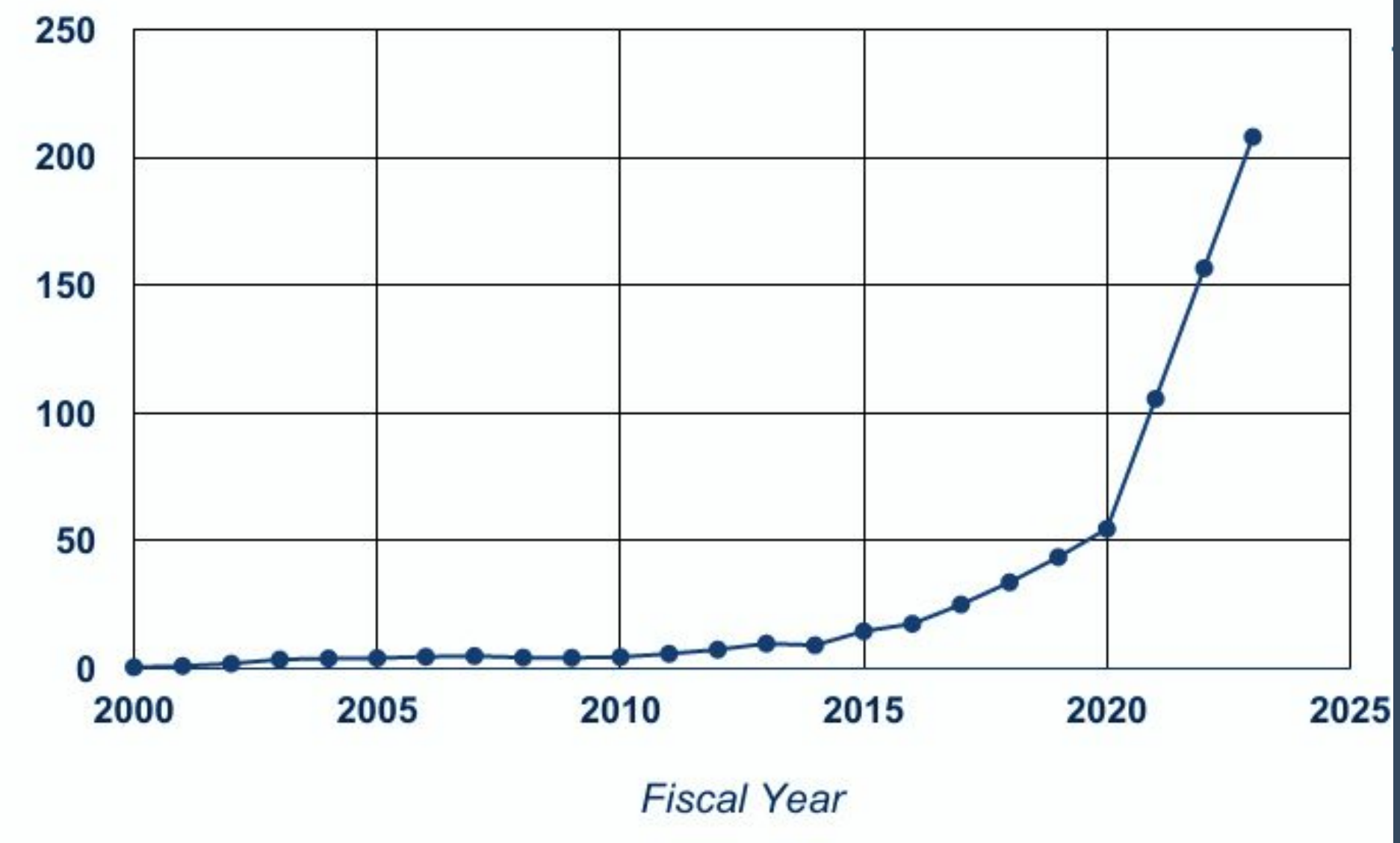



cumulative volume

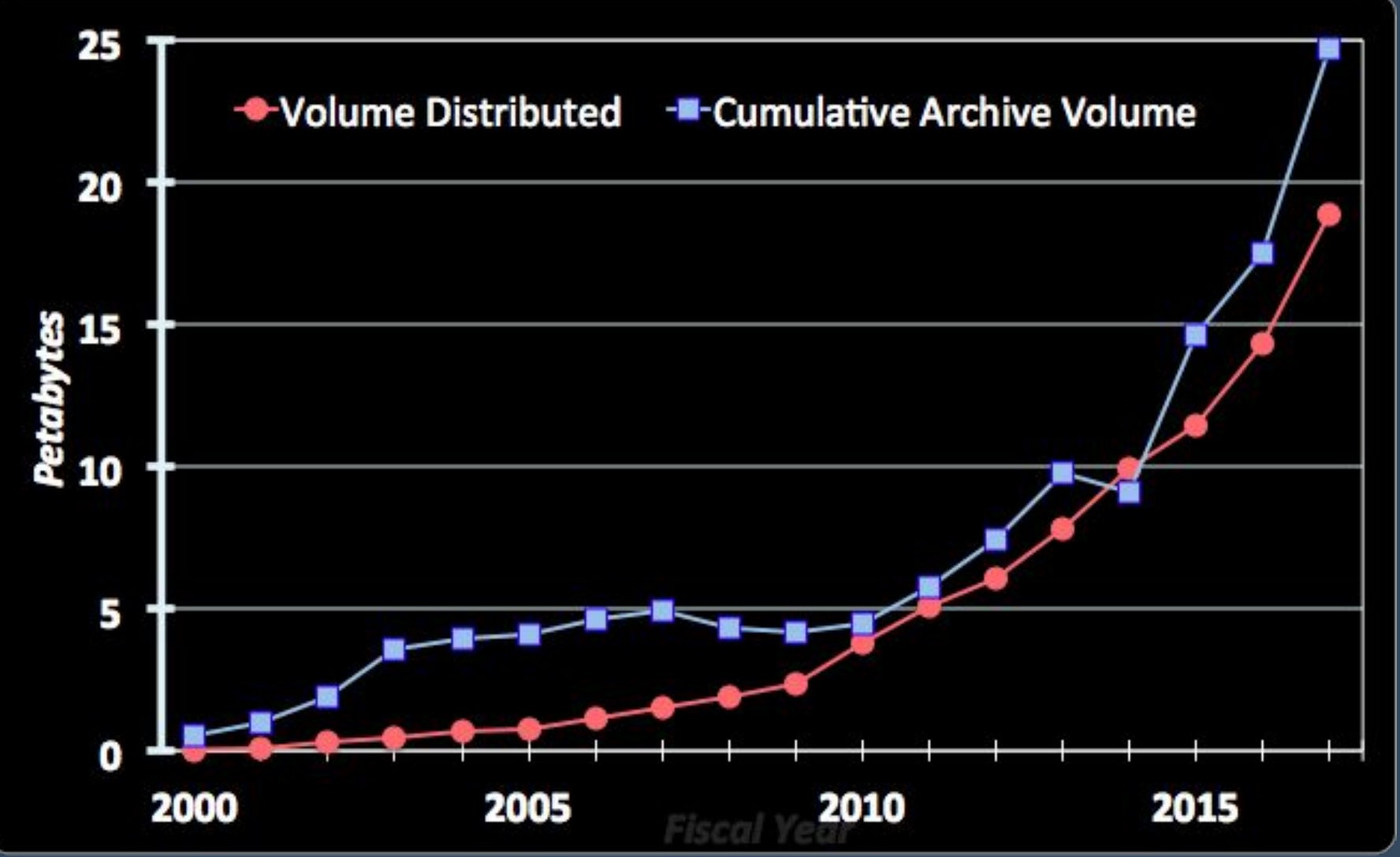


Solution: Data-proximal Analysis

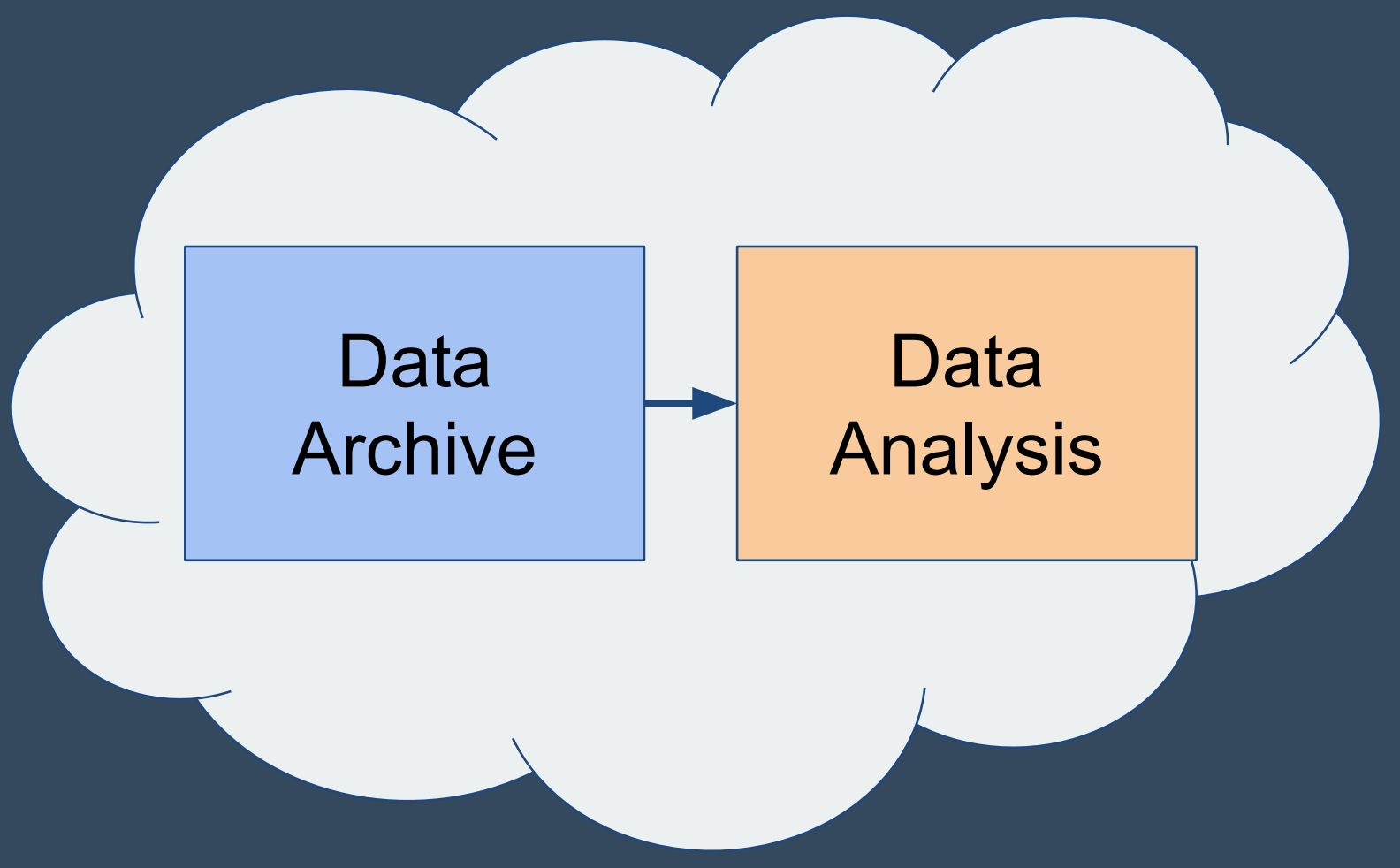




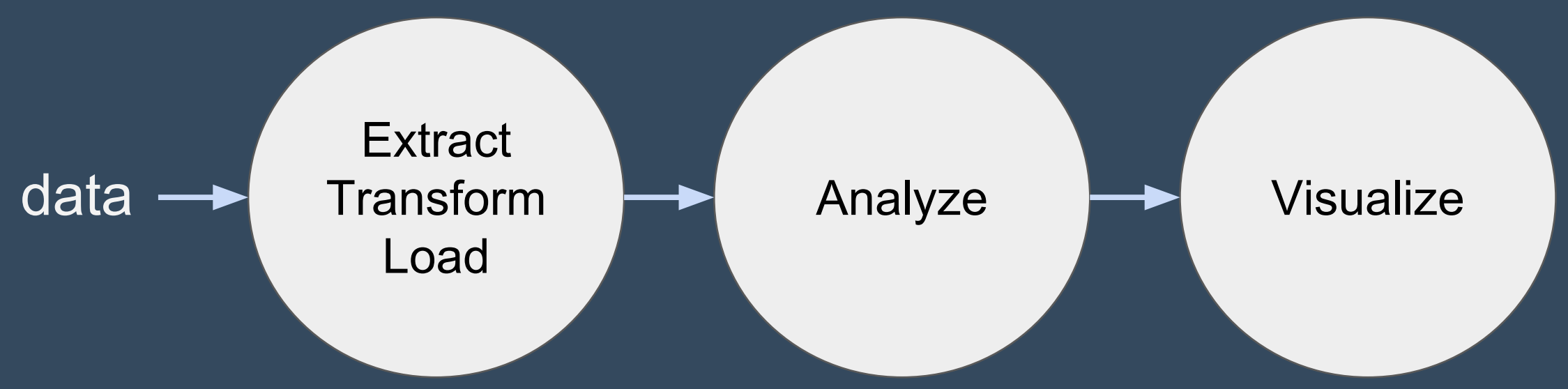




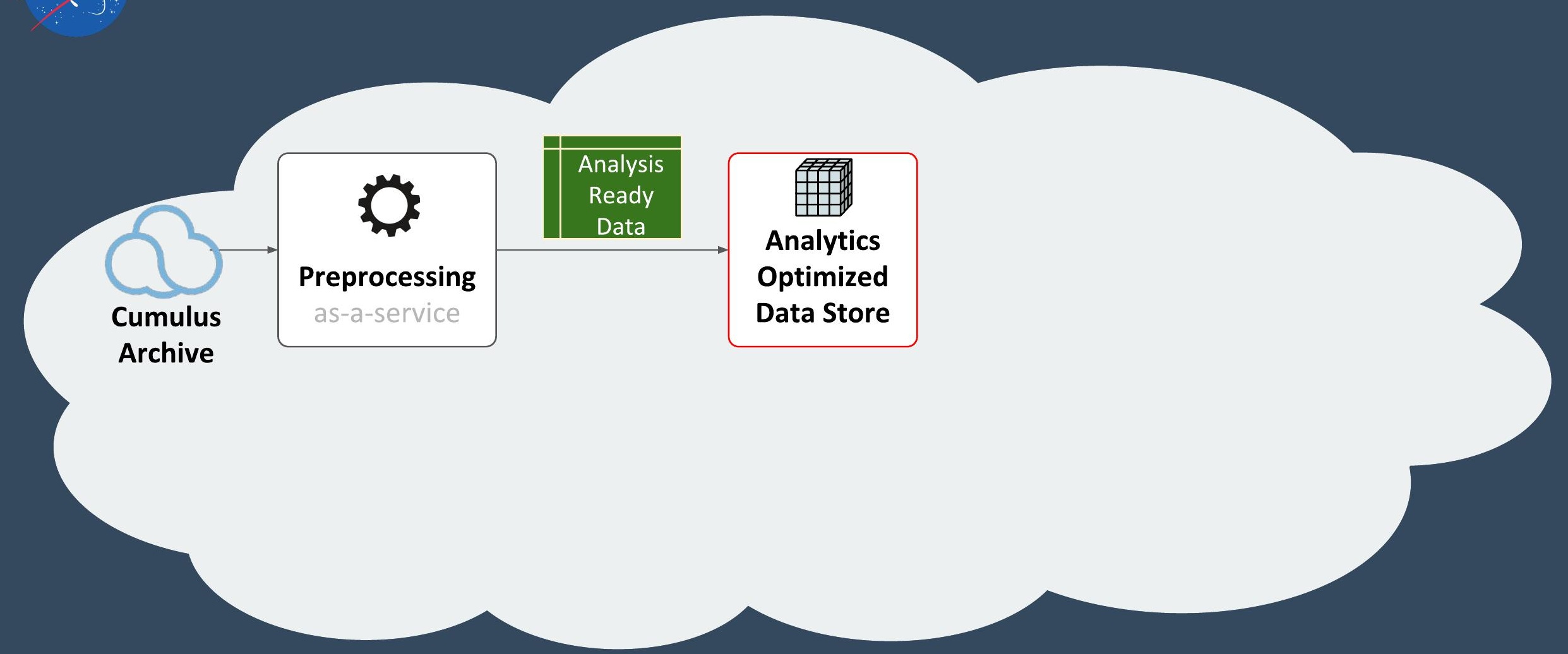




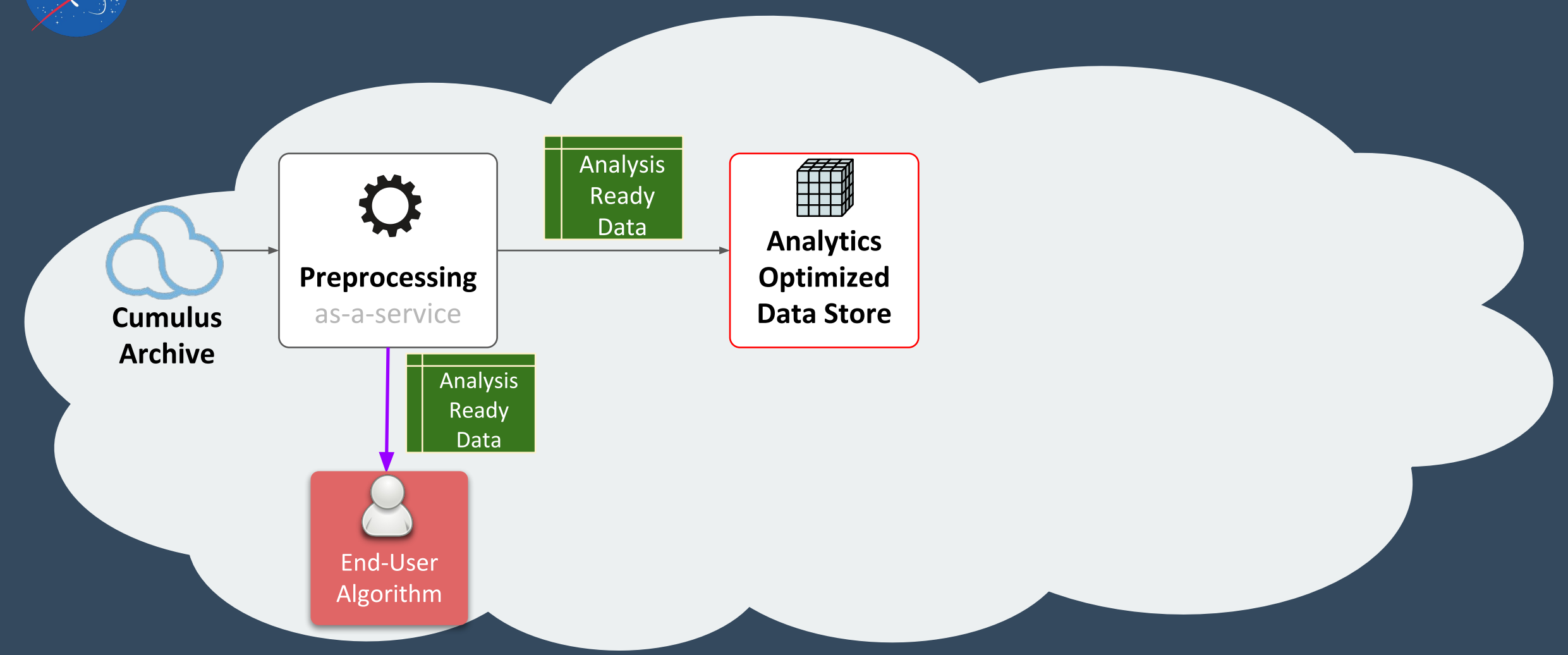




\section{Cloud-Native Analysis for Data Scientists}

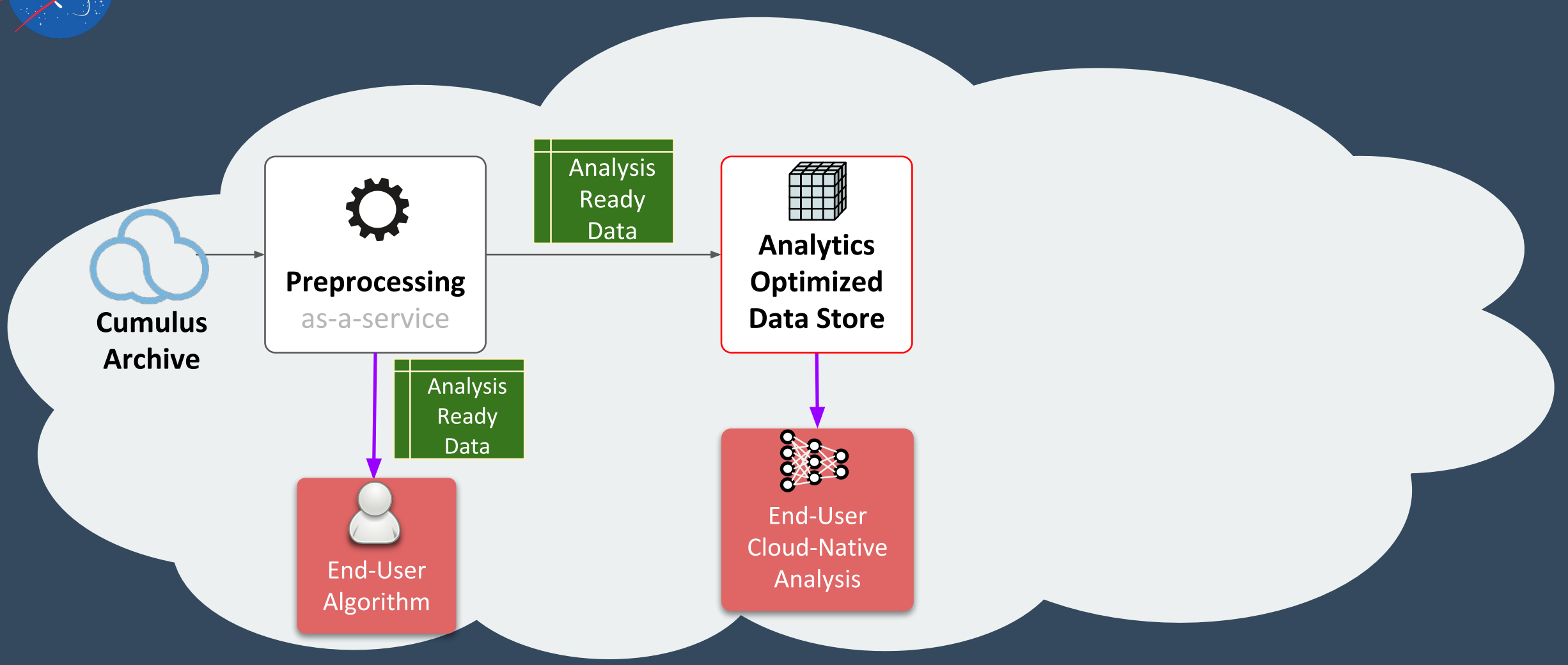




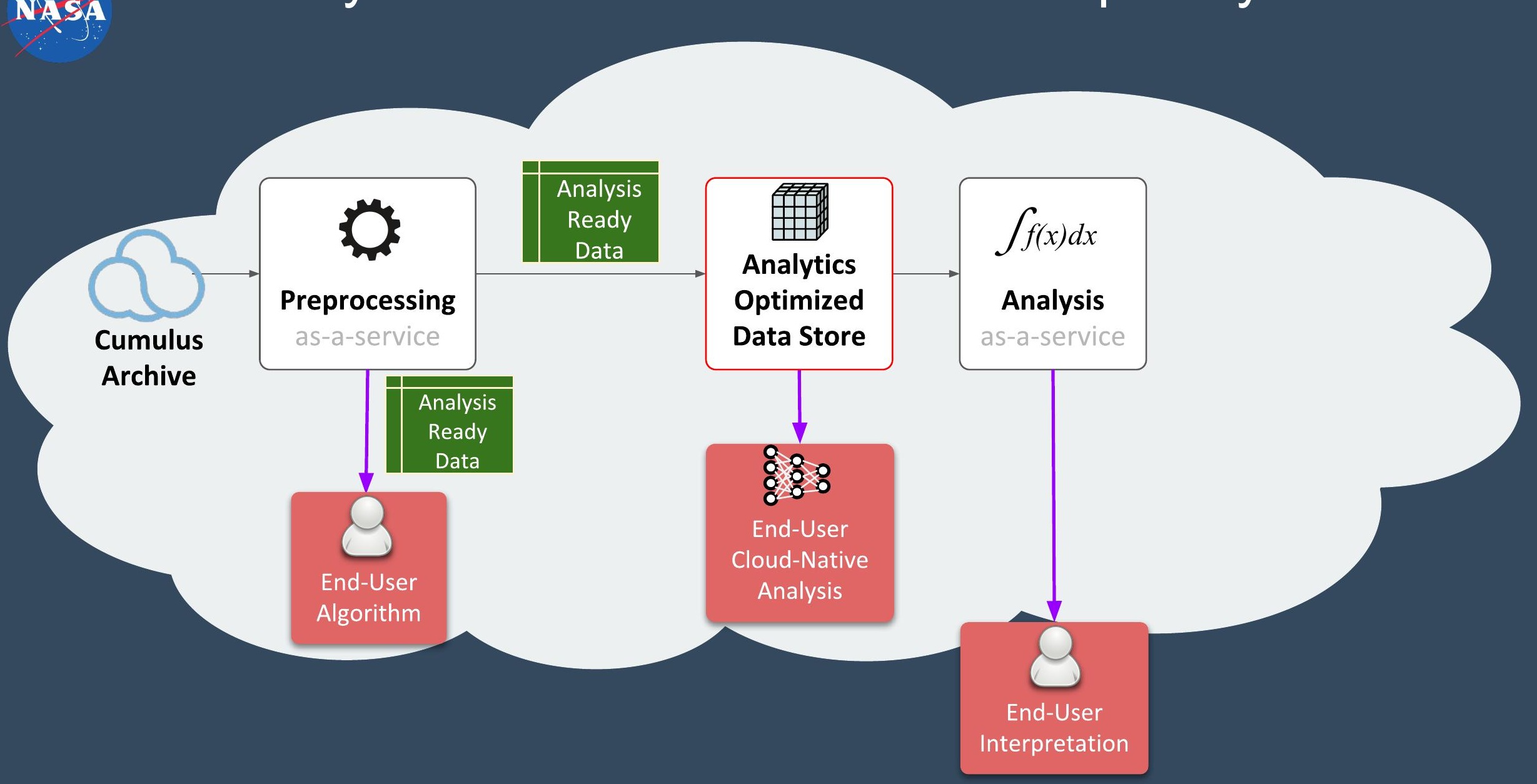




\section{Interactive Data Exploration (for everybody)}

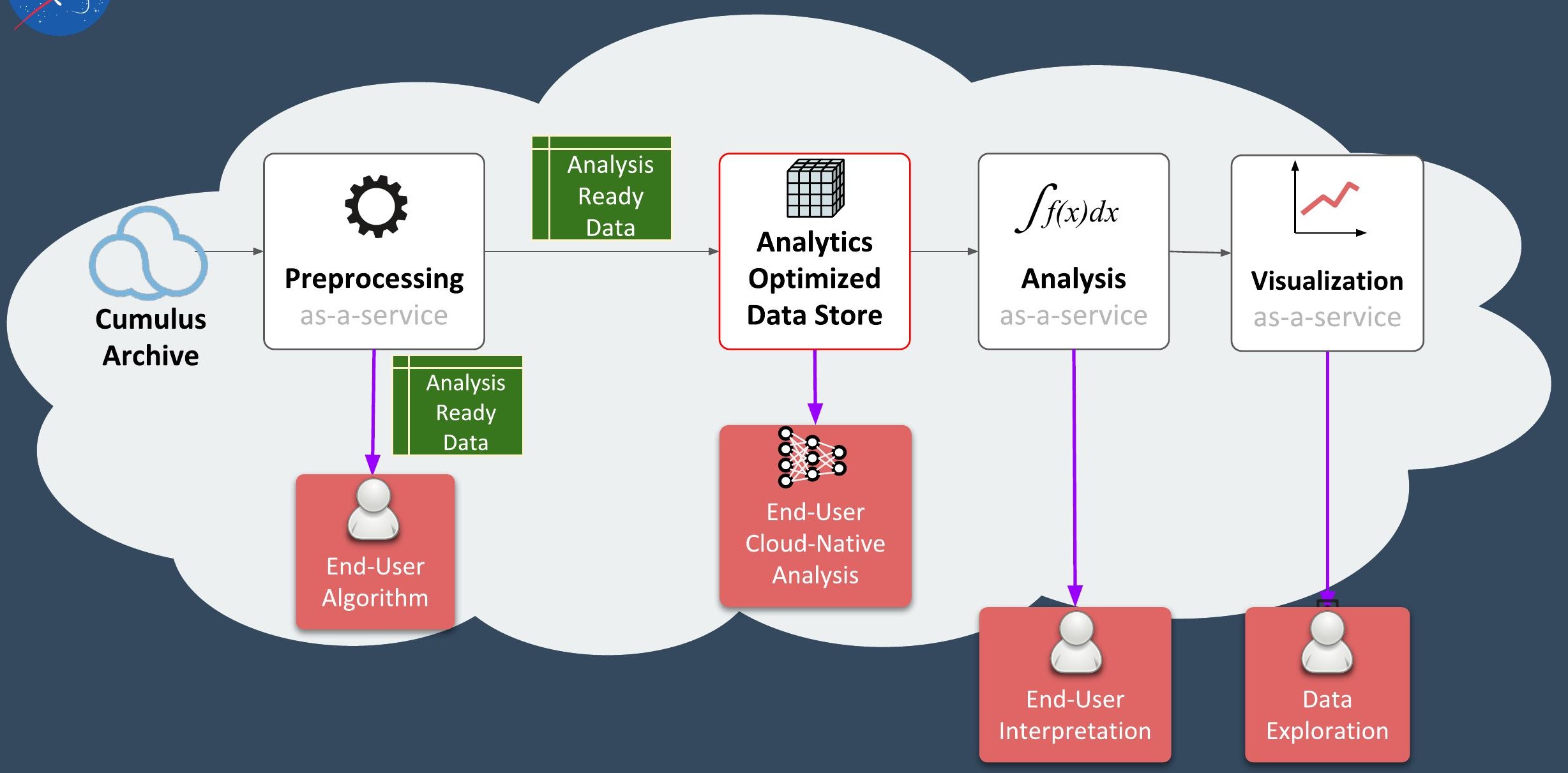


NASA Service offering enables access throughout the value chain

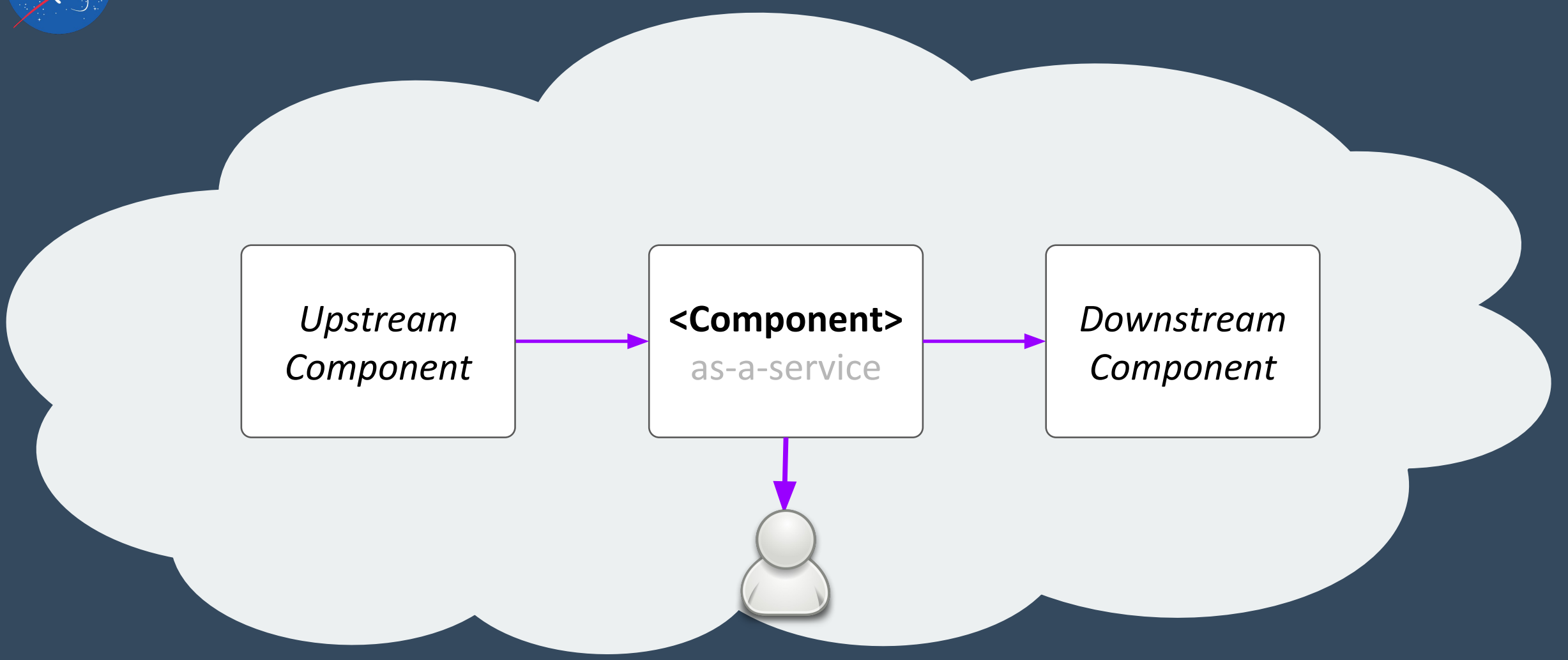




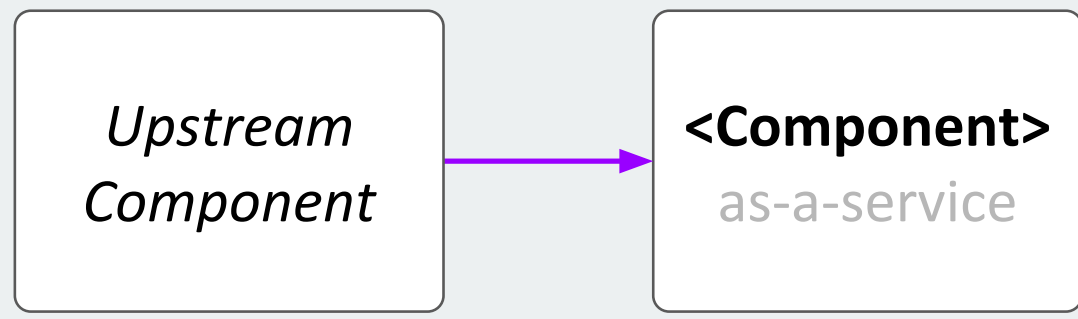


Service consumption opens pipeline to external data

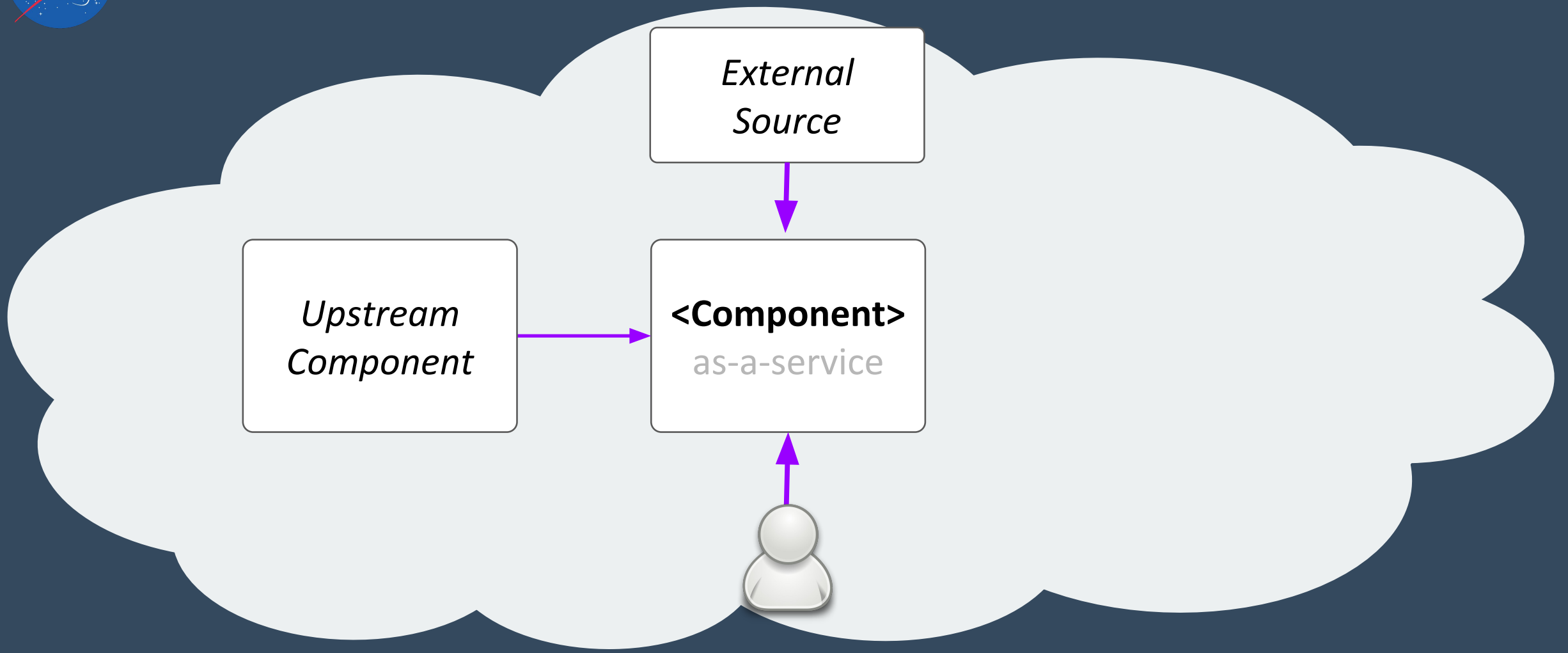


February 2018, Annapolis, Maryland

40 participants:

- Earth scientists

- Policymakers

- System architects

- Data scientists

Report: https://ntrs.nasa.gov/search.jsp?R=20180002954 


\section{Workshop Recommendations}

1. Align Strategies of Cloud Analytic Efforts

2. Develop Reference Architecture for Cloud Analytics

3. Develop Analytics-Optimized Data Stores

4. Enable Reuse of Cloud Analytics-Related Services

5. Foster Wider Machine Learning Adoption...

6. Foster Cloud Adoption 


\section{Key Questions}

Q: What service specifications should components use?

1. High-level: Open Geospatial Consortium family?

a. Web Coverage Processing Service

b. Web Processing Service

2. Low-level: OpenAPI ${ }^{\star}$ ? 


\section{Key Questions}

Q: Which data transformations are common enough to include in production of Analysis-Ready Data?

1. Subsetting

2. Regridding and reprojection

3. Quality filtering? 


\section{Key Questions}

Q: What is the optimal Analytics Optimized Data Store?

1. Highly scalable database?

2. Hadoop File System?

3. Data Cubes in Web Object Storage?

4. Xarray / zarr?

5. It depends... 